\title{
Reacción de reversión que simula erisipela
}

Leprosy type I reaction mimicking erysipelas

\section{María Jimena Latorre-Gómez ${ }^{1}$, Raquel Cristina Maia², Marcos Davi Gomes de Sousa ${ }^{3}$, Brennda Valença2 ${ }^{2}$ Alice Miranda-Machado ${ }^{4}$, Jose Augusto Da Costa Nery ${ }^{5}$}

1. Médica dermatóloga, Instituto Carlos Chagas-Policlínica Geral do Río de Janeiro, Rio de Janeiro, Brasil

2. Médica, Ambulatorio Souza Araújo, Fundación Oswaldo Cruz, Rio de Janeiro, Brasil

3. Medico infectólogo, Instituto Nacional de Infectologia Evandro Chagas; profesor de Enfermedades Infecciosas, Universidad Estácio de Sá, Rio de Janeiro, Brasil

4. Médica patóloga, doctora en Biología Parasitaria, Laboratorio de Lepra; investigadora asociada, Ambulatorio Souza Araújo, Fundación Oswaldo Cruz, Rio de Janeiro, Brasil

5. Médico dermatólogo, magíster en Dermatología, doctorado en Enfermedades Infecciosas y Parasitarias; jefe, Ambulatorio Souza Araújo, Fundación Oswaldo Cruz; jefe, Sector de Dermatología Sanitaria, Instituto de Dermatologia Professor Rubem David Azulay-Santa Casa da Misericórdia do Rio de Janeiro, Brasil; professor titular, Universidade Estácio de Sá; profesor asociado, Policlínica Geral do Rio de Janeiro, Rio de Janeiro, Brasil.

\section{RESUMEN}

La lepra es una enfermedad crónica e insidiosa que puede ser interrumpida por episodios reaccionales, secundarios a una reacción inflamatoria súbita más frecuente en los pacientes con el espectro lepromatoso o dimorfo. Tiene un gran potencial de producir discapacidad y el pronóstico mejora con el control precoz de los episodios, reduciendo el proceso inflamatorio, principalmente aquel que ataca los nervios periféricos cuyo daño ocasiona limitaciones físicas permanentes.

La reacción de reversión (tipo 1) se asocia con un aumento súbito de la inmunidad Th1, caracterizada clínicamente por la exacerbación de las lesiones antiguas o la aparición de otras nuevas.

Se describe el caso de un paciente con lepra multibacilar en tratamiento con poliquimioterapia, que presentaba compromiso sistémico y lesiones eritemato-edematosas con descamación, cuya forma clínica simulaba un cuadro de erisipela. La biopsia y los exámenes complementarios llevaron a concluir que se trataba de una reacción de reversión y se inició el tratamiento específico para el episodio reaccional, obteniéndose una evolución satisfactoria.

PALABRAS CLAVE: reacción de reversión, lepra, erisipela.

\section{SUMMARY}

Leprosy is a chronic insidious disease, sometimes interrupted by reactive episodes, characterized by sudden inflammatory reactions, more frequently affecting lepromatous and dimorphic patients. It shows a high incapacitating potential and prognosis improves with early control of the episodes reducing the inflammatory process that attacks preferably peripheral nerves that causes permanent physical disabilities.
Correspondencia:

Jimena Latorre

Email:

jimenalatorreg@hotmail.com

Recibido: 30/05/2016

Aceptado: 04/06/2017

Conflictos de interés:

No se reportan conflictos de interés.

Financiación:

Ninguna. 
Reverse reaction (type 1) is associated with sudden increase in Th1 immunity, clinically characterized by exacerbation of old lesions or appearance of new ones.

We report a case of reversal reaction affecting a multibacillary patient in treatment with multidrug therapy, with systemic involvement besides erythematous, edematous and scaly lesions, whose atypical clinical presentation resembled erysipelas. Biopsy and complementary tests led us to conclude that it was a reverse reaction and started the specific treatment for the reactional episode with satisfactory evolution.

KEY WORDS: Reversal reaction, leprosy, erysipelas
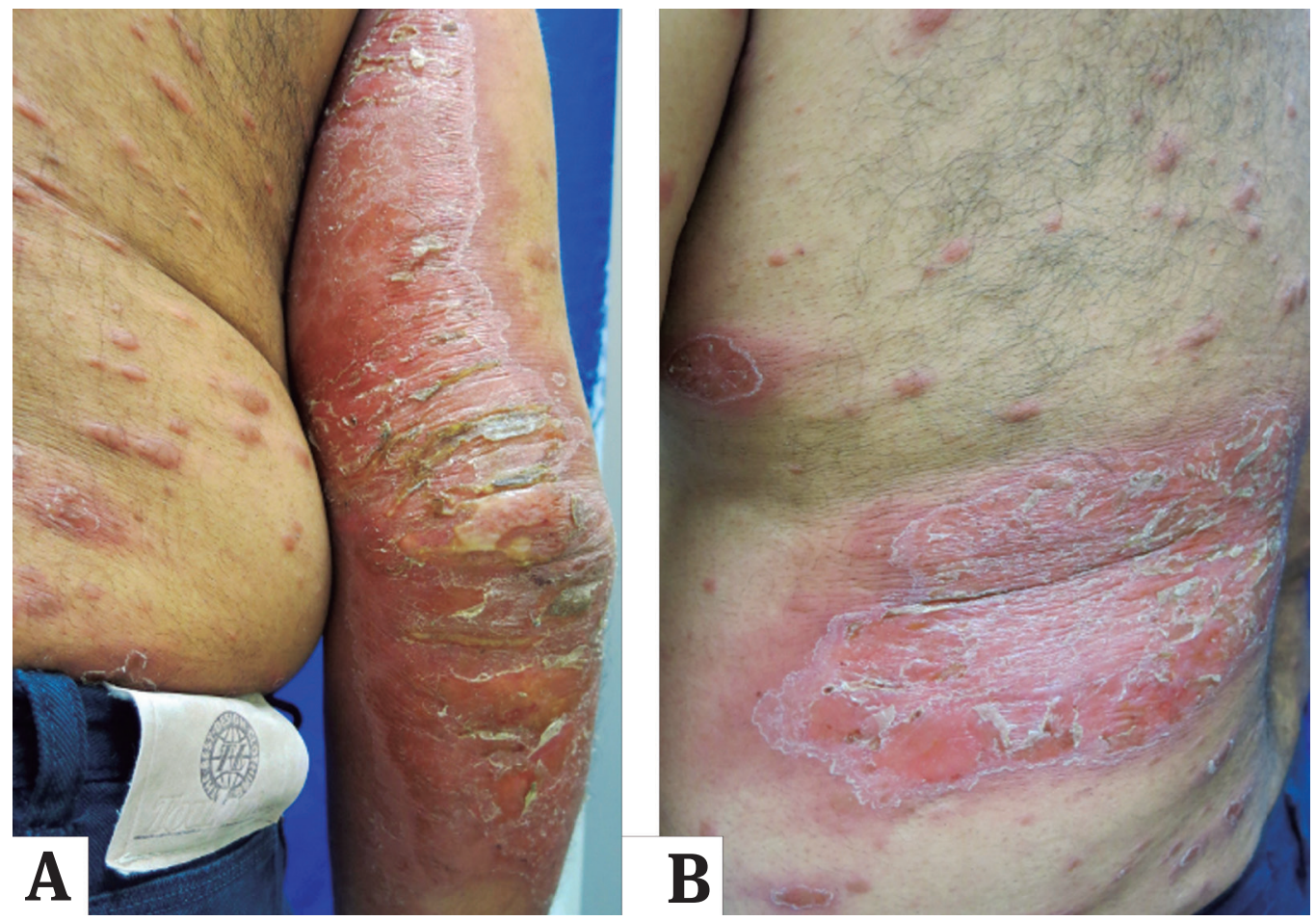

FIGURA 1A Y B. Lesiones eritemato-descamativas, con intenso edema $(+4 / 4)$ y áreas exulceradas

\section{INTRODUCCIÓN}

La lepra es una enfermedad milenaria, considerada un importante problema de salud pública en Suramérica. Después de la India, Brasil es el segundo país con mayor número de casos diagnosticados. El diagnóstico y el tratamiento precoz continúan siendo los desafíos para evitar la discapacidad.

\section{CASO CLIINICO}

Se trata de un paciente de sexo masculino, de 53 años de edad y residente en Río de Janeiro, con diagnóstico de lepra multibacilar y que recibía la décima dosis de poliquimioterapia. Presentó un cuadro clínico de un mes de evolución, de aparición súbita de placas eritematosas asociada a fiebre, mialgias, parestesias, edema, artralgias y alteración en la sensibilidad. No tenía otros antecedentes de importancia. Antes de esta consulta, había recibido $60 \mathrm{mg}$ al día de prednisona y $300 \mathrm{mg}$ al día de talidomida durante 15 días, y una dosis intramuscular única de dipropionato de betametasona.

En el examen físico se encontró un paciente taquicárdico, con más de 20 placas de 8 centímetros, aproximadamente, bien delimitadas, eritemato-edematosas, en la hemicara izquierda, el miembro superior de- 
recho, el dorso posterior derecho, la región interglútea y la cara anterior de las piernas. Presentó importante edema $(+++/++++)$ en el miembro superior derecho y en ambos tobillos (figura 1).

En los exámenes de laboratorio se encontró discreta anemia y macrocitosis, sin leucocitosis. La función renal y la hepática eran normales. La histopatología (figura 2) demostró infiltrado granulomatoso epitelioide maduro, con presencia de células gigantes en la dermis superficial, media y profunda, alrededor de los vasos y los anexos e intraneural. Con la coloración de Wade, no se observaron bacilos ácido-alcohol resistentes (BAAR). El índice logarítmico de la biopsia fue de cero.

Los hallazgos clínicos e histopatológicos fueron indicativos de reacción de reversión (tipo 1). El paciente fue hospitalizado y se administraron pulsos diarios de 1 $\mathrm{mg} / \mathrm{kg}$ de metilprednisolona. Después del tratamiento y por mejoría de la sintomatología, se dio el alta hospitalaria (figura 3) y se continuó el tratamiento para lepra multibacilar.

\section{DISCUSIÓN}

La lepra es una enfermedad infectocontagiosa, granulomatosa y crónica, causada por Mycobacterium leprae y caracterizada por alteraciones neurocutáneas, cuya transmisión se produce por contacto con aerosoles de las vías aéreas superiores. Brasil, después de la India, es el segundo país con mayor prevalencia; se considera una enfermedad endémica muy infecciosa y poco patogénica ${ }^{1}$.

Se clasifica según criterios clínicos, histopatológicos, inmunológicos y bacteriológicos. La primera clasificación descrita en Madrid en 1953 incluye dos tipos polares: lepromatoso y tuberculoide, así como dos grupos de casos: uno de principio de la lepra (casos indeterminados) y casos de inmunología dudosa (dimorfos o interpolares [borderline]). Posteriormente, en 1966, Ridley y Jopling subdividieron el grupo dimorfo según la forma en la que se comporta la infección en el espectro inmunológico: BT si se encuentra cerca del polo tuberculoide, BL cuando está cerca del polo lepromatoso y BB si se localiza en el centro de este espectro.

Los períodos de agudización de la lepra, conocidos como reacciones, son consecuencia del crecimiento bacilar capaz de romper las células y desencadenar reacciones celulares y humorales que se correlacionan clínicamente con la fiebre y el mal estado general, pudiéndose presentar antes, durante o después del tratamiento como reacción a mecanismos inflamatorios o como reacción inmunológica al tratamiento, con presencia de bacilos fragmentados o gránulos con potencial antigénico ${ }^{3}$.

La reacción de tipo 1 (habitual en los casos tuberculoides y dimorfos, y rara en los lepromatosos es mediada por la inmunidad celular, siendo ejemplo típico de las reacciones de hipersensibilidad tardía in vivo, reacción de tipo IV de Gell y Coombs. Las lesiones cutáneas ya existentes se convierten en lesiones eritema-

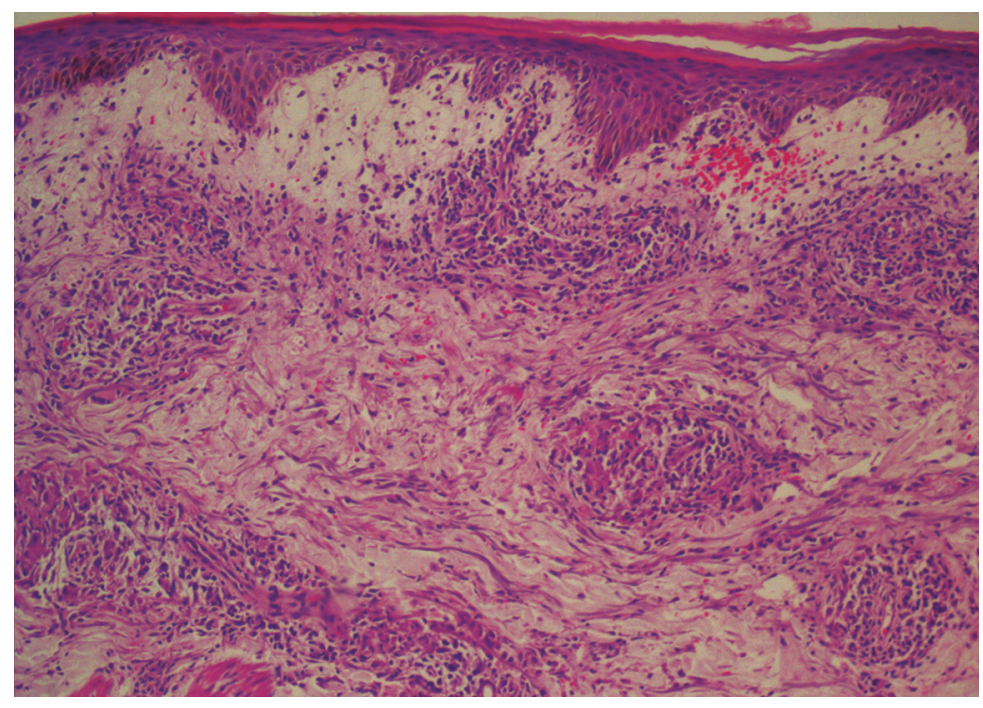

FIGURA 2. Infiltrado granulomatoso epitelioide maduro con abundancia de linfocitos y presencia de edema importante en la dermis papilar, células gigantes en la dermis superficial, media y profunda, perivascular, perianexial e intraneural. Hematoxilina y eosina, 2oX. 

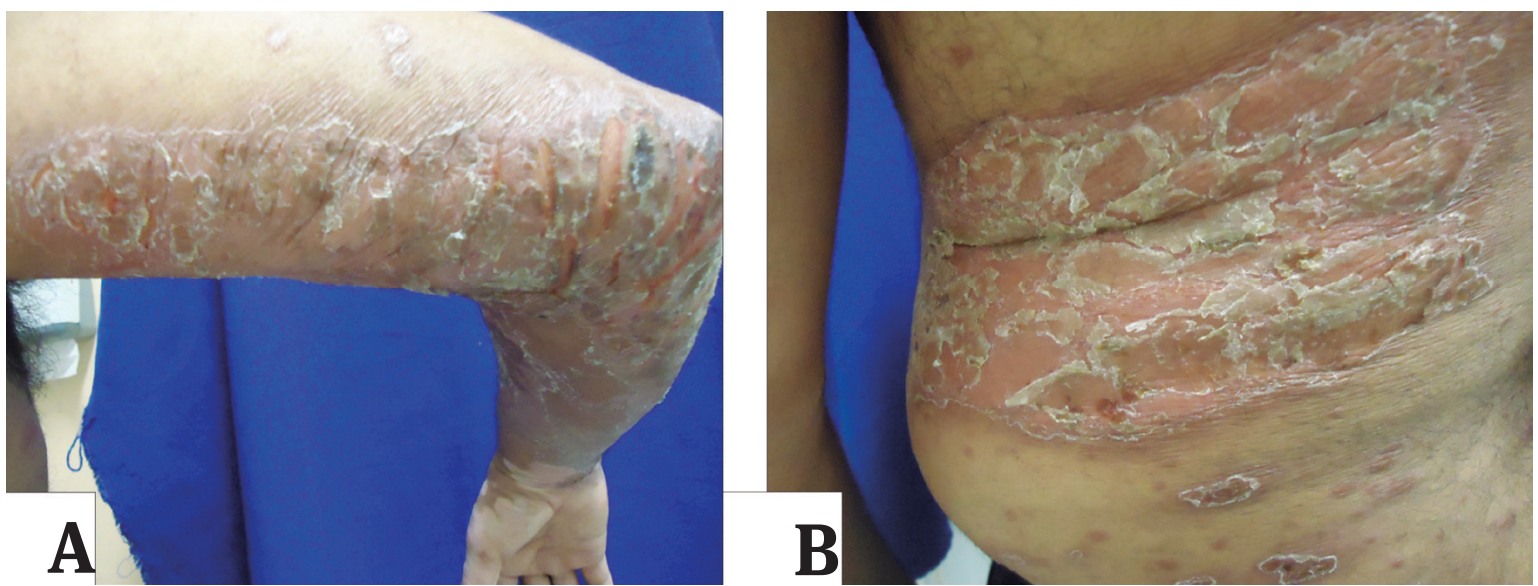

FIGURA 3A Y B. Placas con descamación de bordes definidos, en resolución

tosas, edematosas, brillantes, infiltradas, con borde mejor delimitado y con alteración en la sensibilidad 4 . Es frecuente la neuritis aguda, principalmente en los nervios cubital, mediano, radial, peroneo, tibial posterior y facial. La lesión neural puede afectar las fibras sensitivas y motoras de los troncos nerviosos periféricos, generando lesiones definitivas y discapacidad física ${ }^{5}$.

La reacción de tipo 2, o eritema nudoso lepromatoso, típica del paciente multibacilar, es mediada por la inmunidad humoral. Representa una reacción mediada por complejos inmunes circulantes, también llamada de tipo III en la clasificación de Gell y Coombs, y se caracteriza por la aparición de nódulos eritematosos dolorosos que pueden ulcerarse, principalmente en los miembros inferiores. Además, hay compromiso del estado general, fiebre, toxemia, mialgias, artritis, iritis, epididimitis, orquitis y neuropatía ${ }^{6}$. En la tabla 1 se presentan algunas diferencias entre los dos tipos de reacciones lepromatosas.

Con el curso de la enfermedad, las lesiones de la reacción de reversión se convierten en acastañadas e infiltradas y, posteriormente, evolucionan a lesiones con descamación o maculares hipocrómicas, que pueden o no pigmentarse con el tiempo. Además, pueden aparecer súbitamente nuevas lesiones con iguales características, frecuentemente en el sexto mes de la poliquimioterapia.

En la histología se observa edema intersticial e intracelular, con aumento de linfocitos, células epitelioides, gigantes y de los granulomas, y disminución de los bacilos, lo cual indica la actividad de la reacción en la eliminación de los mismos ${ }^{7}$. Los estados de inmunosupresión, el embarazo, las enfermedades concomitantes o las infecciones recurrentes, pueden desencadenar cuadros reaccionales. En las reacciones graves con daño extenso del tegumento, las lesiones pueden presentar edema importante, ulceración y necrosis, que simulan una erisipela ${ }^{4}$.

Casos como el descrito en este artículo, con gran compromiso cutáneo y sistémico, se convierten en desafíos diagnósticos y pueden ser mal diagnosticados pues simulan otras enfermedades dermatológicas más frecuentes en la práctica clínica ${ }^{8}$, como la erisipela $\mathrm{u}$ otras causadas por Staphylococcus aureus o por Streptococcus pyogenes y podrían recibir un tratamiento inadecuado. Por esta razón, se hace imprescindible una anamnesis y un examen físico minuciosos.

La erisipela es un proceso cutáneo superficial, generalmente propio de la dermis aunque con prominente compromiso linfático y diferente clínicamente de otras infecciones cutáneas básicamente por tres aspectos: las lesiones son elevadas, existe una clara línea demarcada entre la piel afectada y el tejido sano, y las lesiones son clásicamente eritematosas y de color salmón 9 .

La reacción reversa que simula erisipela, tiene como tratamiento de primera línea la metilprednisolona en dosis diarias de $1 \mathrm{mg} / \mathrm{kg}$, la cual ayuda a disminuir la inflamación y el posible daño neural, con disminución gradual según la evolución de las lesiones; el promedio de tratamiento es de cuatro a nueve meses. La ciclosporina y la azatioprina son el tratamiento de segunda línes cuando el corticoide está contraindicado ${ }^{10}$. 


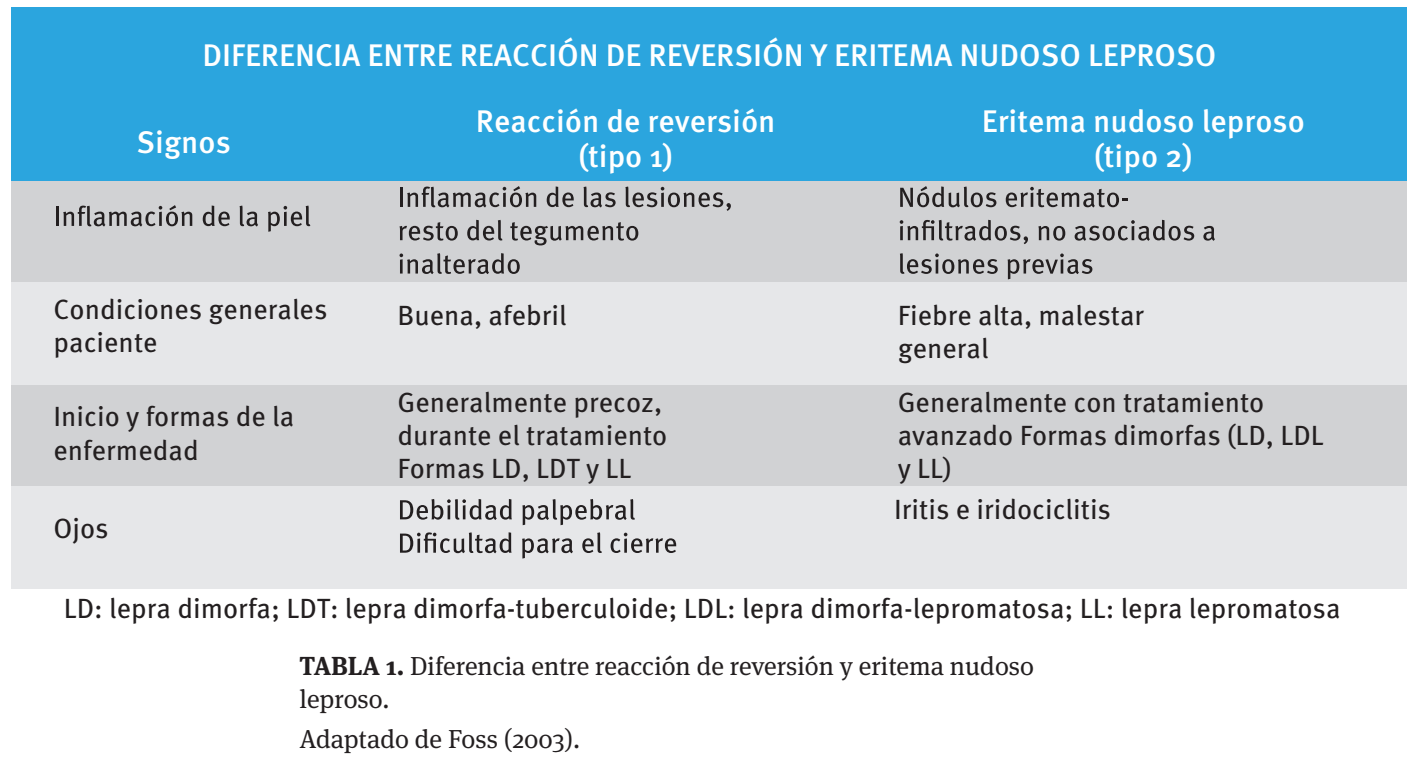

\section{CONCLUSIÓN}

La lepra es una enfermedad crónica, contagiosa y curable, con periodos de mayor compromiso neurocutáneo llamados reacciones, que pueden producir un alto grado de discapacidad motora y sensitiva, y que generan discriminación.

Son raros los casos reportados en la literatura científica de reacción de reversión que simulan erisipela. El no reconocer esa forma de presentación podría conducir al uso innecesario de antibióticos y retardar el inicio del tratamiento.

El tratamiento con pulsos de metilprednisolona se ha empleado en el control de los episodios reaccionales graves o con mala reacción terapéutica y en otras indicaciones que mejorarían la supervivencia del paciente. El diagnóstico correcto y el tratamiento precoz son fundamentales para el control de la enfermedad y la eliminación del estigma, cuyas repercusiones sicológicas ocasionadas por las secuelas físicas son factores que influyen en la autoestima y en la discriminación del paciente.

\section{REFERENCIAS}

1. Araujo MG. Hanseníase no Brasil. Rev Soc Bras Med Trop. 2003;36:373-82.

2. Azulay RD, Azulay DR, Azulay L. Dermatología. Quinta edição. Rio de Janeiro: Editorial Guanabara Koogan; 2011. p. 321-37.

3. Foss NT. Episódios reacionais na hanseníase. Me- dicina (Ribeirão Preto). 2003;36:453-9.

4. Souza CS. Hanseníase: formas clínicas e diagnóstico diferencial. Medicina (Ribeirão Preto). 1997;30:325-34.

5. Naafs B, van Hees C. Leprosy type 1 reaction (formerly reversal reaction). Clin Dermatol. 2016;34:37-50.

6. Opromolla DV. Manifestações clínicas e reações. Em: Opromolla DVA, editor. Noções de hansenologia. Bauru: Centro de Estudos Dr. Reynaldo Quagliato; 2000. p. 51-8.

7. Alves ED, Ferreira TL, Nery I. Hanseníase: avanços e desafios. Brasilia, D.F.: Universidade de Brasília; 2014. p. 189-215.

8. Nery JA, Sales AM, Illarramendi X, Duppre NC, Jardim MR, Machado AM. Contribuição ao diagnóstico e manejo dos estados reacionais. Uma abordagem prática. An Bras Dermatol. 2006;81:367-75.

9. Ferretti JJ,. Stevens DL, Fischetti VA.. Streptococcus pyogenes: Basic biology to clinical manifestations Oklahoma City, OK: Ed. University of Oklahoma; 2016. p. 544-52.

10. Sociedade Brasileira de Hansenologia, Academia Brasileira de Neurologia e Sociedade Brasileira de Neurofisiologia Clínica. Hanseníase: diagnóstico e tratamento da neuropatia. Projeto Diretrizes, Associação Médica Brasileira e Conselho Federal de Medicina; 2003. Disponible en: https://diretrizes. amb.org.br/_BibliotecaAntiga/hanseniase-diagnostico-e-tratamento-da-neuropatia.pdf. 